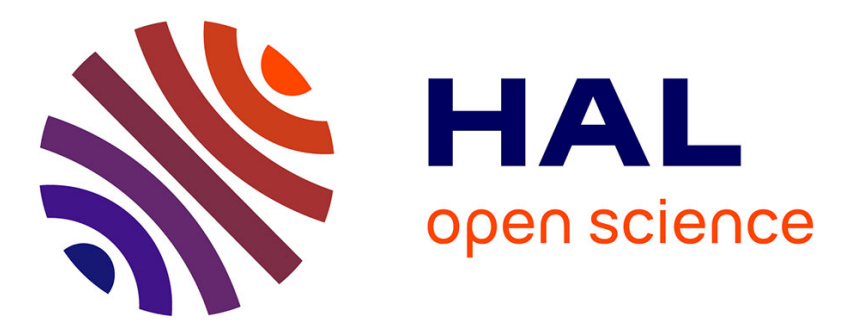

\title{
Secure and Internet-Less Connectivity to a Blockchain Network for Limited Connectivity Bank Users
}

Daniel Maldonado-Ruiz, Mohamad Badra, Nour El Madhoun, Jenny Torres

\section{To cite this version:}

Daniel Maldonado-Ruiz, Mohamad Badra, Nour El Madhoun, Jenny Torres. Secure and InternetLess Connectivity to a Blockchain Network for Limited Connectivity Bank Users. MSPN 2020: International Conference on Mobile, Secure and Programmable Networking, Oct 2020, Paris, France. hal-02972585

\section{HAL Id: hal-02972585 \\ https://hal.science/hal-02972585}

Submitted on 20 Oct 2020

HAL is a multi-disciplinary open access archive for the deposit and dissemination of scientific research documents, whether they are published or not. The documents may come from teaching and research institutions in France or abroad, or from public or private research centers.
L'archive ouverte pluridisciplinaire HAL, est destinée au dépôt et à la diffusion de documents scientifiques de niveau recherche, publiés ou non, émanant des établissements d'enseignement et de recherche français ou étrangers, des laboratoires publics ou privés. 


\title{
Secure and Internet-Less Connectivity to a Blockchain Network for Limited Connectivity Bank Users
}

\author{
Daniel Maldonado-Ruiz ${ }^{1}$, Mohamad Badra ${ }^{2}$, Nour El Madhoun ${ }^{3}$, and Jenny Torres ${ }^{1}$ \\ 1 Departamento de Informática y Ciencias de la Computación, \\ Facultad de Ingeniería en Sistemas Informáticos y Computación, \\ Escuela Politécnica Nacional, Ecuador \\ \{daniel.maldonado02, jenny.torres\}@epn.edu.ec \\ 2 College of Technological Innovation, Zayed University, P.O. Box 19282 Dubai, U.A.E \\ mohamad.badra@zu.ac.ae \\ 3 Security and System Laboratory, EPITA, 14-16 Rue Voltaire 94270 Le Kremlin-Bicêtre, France \\ nour.el-madhoun@epita.fr
}

\begin{abstract}
Over the past few years, we have seen the emergence of a wide range of banking architectures, technologies, and applications made possible by the significant improvements in hardware, software, and networking technologies. Nowadays, innovative solutions are being developed by banks to leverage the benefits of blockchain, to improve their business agility and performance, and to make their business operations more efficient and secure. However, there are still cases where regular access to Internet is impossible or unreliable due to saturated networks or harsh environments, hence limiting the deployment of typical blockchain based solutions. In this context, an approach using a new connectivity technology is needed in order to increase mobile Internet services for any device to reach nearly $95 \%$ of the world population, instantly, simply by drawing on existing mobile phone networks, with no additional infrastructure development. We aim to give the user full bank access from their device, even if the device is not a smart one, using ordinary mobile phone networks. However, providing efficient and secure communications over lossy and low bandwidth networks remains a challenge. The main objective of this paper will be to design an end-to-end and low overhead secure solution for the communications between mobile devices and their corresponding remote application servers that using blockchain via ordinary mobile networks.
\end{abstract}

Keywords: banking transactions $\cdot$ offline networks $\cdot$ mobile networks $\cdot$ SMS $\cdot$ blockchain $\cdot$ Ethereum - Smart Contracts.

\section{Introduction}

Mobile Internet access and mobile transactions have become the base of modern communications between any kind of mobile devices, be these classic ones or IoT. The users of mobile Internet can interact with their applications and systems in a variety of ways and in real time. Using mobile Internet to access banks is not worth the risk, time or costs. However, mobile banking has greater flexibility and convenience because transactions can be done anywhere, anytime, and using any mobile device. It is therefore in the interest of banks and their customers to find a way to make mobile banking safe and secure.

We are currently witnessing the adaptation of a blockchain system to explore new methods to effectively manage the security aspects of applications and services, to assure data transparency, to ensure cost efficiency when managing, and data processing. Innovative solutions are being developed by enterprises and institutions to leverage the benefits of the blockchain, to improve their business agility and performance, and to make their business operations more efficient and secure.

Blockchain is basically known as the technology behind the Bitcoin cryptocurrency [1] [2]. It is a distributed/shared public ledger with a set of specific predefined rules that determine how the ledger is appended by the distributed consensus of the participants in the network. It offers a new paradigm for implementing transactions in a trust-less environment, where participants agree on the validity of the transactions that are recorded in the distributed ledger. The ledger is only updated by consensus of the participants and each transaction recorded in the ledger will be cryptographically verifiable with proof of agreement from the participants. As a result, each participant will have a copy of the same ledger with records that are 
immutable, so they cannot be altered or deleted later. In this way, blockchain eliminates the need for central authorities, making asset provenance and traceability more efficient and transparent than existing systems. Existing Blockchain platforms, such as Ethereum [3] or Hyperledger blockchain technologies, enable the use of smart contracts to digitalize and self-execute contractual agreements between different parties without the need for any central authority. Smart contracts are defined as self-executing contractual states automating the execution of business workflows in a fully decentralized and trust-less blockchain. However, having access to a stable Internet connection is a prerequisite to enable the deployment of blockchain based applications.

Although $95 \%$ of the world population has access to to all kinds of mobile phones, in developing countries, like those in Africa or Asia for instance, $75 \%$ of people do not have access to Internet. Also, there are still cases in those countries where regular access to Internet is impossible or unreliable due to saturated networks or harsh environments, hence limiting the deployment of any Blockchain based solution. In this context, we propose a solution that aims at increasing mobile Internet services for devices that do not have access to Internet in the first place. The main idea is to reach nearly $95 \%$ of the world population, instantly, simply by drawing on existing mobile phone networks (GSM / 2G), with no additional infrastructure development. The main objective of our paper will be to design an end-to-end and low overhead security solution to allow the communications between a mobile devices and their corresponding remote applications servers for GSM users not having access to mobile Internet. Our proposed solution creates a new way to extend centralized systems, such as banks and financial systems, to leverage decentralized transaction systems and decentralised identity validators. In particular, it aims to a) secure transactions between users and entities, and b) create new ways to enable these transactions for vulnerable users, which can use the system by a symplified user validation over a challenge-response validation system. It also aims to integrate the blockchain to record data that should be safe, auditable and resistant to outages.

However, providing efficient and secure communications over lossy and low bandwidth networks remains a challenge, specially in regions without a stable mobile network coverage. Addressing not only any possible delay on communication but network tampering is part of the analysis in order to find the possible solutions to build a secure transaction system for non-smart mobile devices.

The rest of this paper is organized as follows. Section 2 presents the relevant known works about banking communications using mobile networks and blockchain, focusing on systems where broadband communications are limited. We describe our privacy-preserving approach and design in Section 3, followed by its security analysis and performance evaluation in Section 4. Finally, we conclude this paper in Section 5.

\section{Related Work}

Several solutions have been proposed and deployed to enable financial transactions and financial technologies over decentralized ledgers [4]. In 2008, a Payment Application Data Security Standard (PA-DSS) was developed to standardize the security requirements of financial transactions. Applying it to Ethereum, Bello et al. [5] proved that, focusing on business operations, the ledger has a lot of security relying on the storage and implementation aspects, but fails on providing privacy of the transactions and information stored on the ledger. $\mathrm{Xu}$ et al. [6] propose a layered model based on Smart Contracts to ensure the access to the Open banking paradigm, where the smart contract manages the transaction but does not perform it. In cloud systems, Oktian et al. [7] describe a system where users can request information from the cloud. For that research, smart contracts are used to register an account into the cloud by the user before starting any transaction. To ensure the transaction's honesty, each user must pay a fee to maintain the subscription, so malicious subscribers can be avoided. Another approach proposed by Bhaskaran et al. [8] replaces the cloud with two blind entities (financial providers) to manage and validate the smart contracts for every user in the system. The security is defined by a double validation to create a wider network for the blockchain and to secure the information stored on the Smart Contracts. Similar approaches are used to maintain credit reporting [9] and cryptocurrency wallet management [10]. All of these solutions, however, maintain the idea of using the blockchain in a online environment, where all actors must have a broadband access. Having a broadband access allows users to use any modern device to access the system, which is not the idea proposed in this paper, where we focus on users who do not have the ability to have a running smart device that is equipped with a stable broadband connection. In opposite to the previous works, Hu et al. [11] describe a scenario where a community-run base station provides reliable local network connectivity while intermittently connecting to the broader Internet. In this scenario, users depend on smart devices to become miners 
or use some lightweight wallet to generate all transactions to be stored on a Ethereum blockchain with an access control that is based on tokens acting as currency intermediaries. This research incorporates the idea of using mobile devices to connect nodes to a blockchain. However, the proposal still depends on a broadband access and a smart device to perform the transactions, which is very difficult to find in harsh environments.

\subsection{Blockchain Technology}

Blockchain could be defined as a tamper-resistant distributed data ledger, where all the cells are linked by the hash of the previous one, turning the ledger into a "chain of blocks" [12] [13]. As a tool, blockchain exists since 2008 with Nakamoto's Bitcoin, but as a technology could be tracked since 1991 [14]. The main features of blockchain are that offers a decentralized and distributed storage system that doesn't require any third party validation to assure the stored information.

Part of the current blockchain hype lies on the immutability of its structure, because all the records stored on every block cannot be tampered without alter all the blockchain itself, making the system very secure to use in all kinds of transactions.

To store the data, blockchain uses algorithms which allow to create new blocks in the system that synchronize with the entire network in a unique way: the consensus algorithms. These algorithms aimed to create an environment where only the correct block will be store in the ledger, avoiding apocryphal or malicious branches. In Bitcoin, the way to store a new block is made by a process called mining, where a set of nodes called miners try to solve a puzzle in order to find the correct new block. When the new block is found, it is broadcasted to all network, eliminating all additional possible branches. This kind of algorithms where any node could participate in the search of the new block are part of the so-called permissionless (public) blockchain. On the other hand, the algorithms where only a few nodes have the full copy of the ledger and could create the new blocks (with different consensus algorithms) are called permissioned (private) blockchain. Ethereum and Hyperledger are the best known permissioned blockchain applications.

Blockchain Applications Besides the cryptocurrency and smart contracts applications of the ledger, there are some other implementations of blockchain, appeared in recent years. One of the main uses for blockchain is with Internet of Things (IoT) [15] [16], because the ledger can store not only identities of the devices but other features that are used to analyze the behaviour of the 'things'. In that case, blockchain is a strong ally of Big Data [17] [18] [19], because can store a lots of information, either raw or through smart contracts, making the analysis easier and more trustable. Most recent applications of blockchain are a core system to implement DNS and PKI systems [20] [21] [22] [23], where the data could be stored and accessed in a easy way. Other field where blockchain is used these days is to control e-voting systems [24] [25] [26], where the ledger offers a solution of electoral fraud and also offers all the auditory measurements that a system needs. However, this field has security and trust issues in its implementation, specially in developing countries [27]

\section{System design}

Our proposed transaction model enables an Internet-less link between banking users and financial institutions and creates communication channels allowing offline users, (i.e., users with no Internet access or any broadband connection) to perform transactions.

Our proposal, unlike traditional Bitcoin/Ethereum transactions, requires an origin and a destination bank account instead of a user identification, since the entire transactional management will be purely virtual due to the obvious impossibility of transmitting physical money via SMS (Short Message Service). Consequently, every user identity will be managed by the bank, using the registered user account and and the identity information related to it.

To better explain the features of our proposal, we first describe the communication system structure, then we discuss our proposal's communication features and specifications.

\subsection{Communication System Structure}

Fig. 1 illustrates the structure of our proposed approach. First, each user should register to the offline system using his bank account credentials. Hence, the users' identity will always be managed by the bank. The 
registration allows the system to create a smart contract, which contains the user ID (some identification related to the user's bank data), the balance account of the user and a transaction log. Next, a specific mnemonic ASCII PIN will be generated for each registered user, by using both the smart contract and the user ID. The mnemonic ASCII PIN will be used to validate the user to the mobile network. The generation of this PIN emulates the creation of a wallet address on a Bitcoin wallet [1]. Even though transactions are made in a cellphone, the phone number is not an important component here. With the mnemonic ASCII PIN, users can access to the transaction system from any cellphone, because the validation is always made through that PIN. In our model, all messages exchanged between the user and the system are transported by SMS.

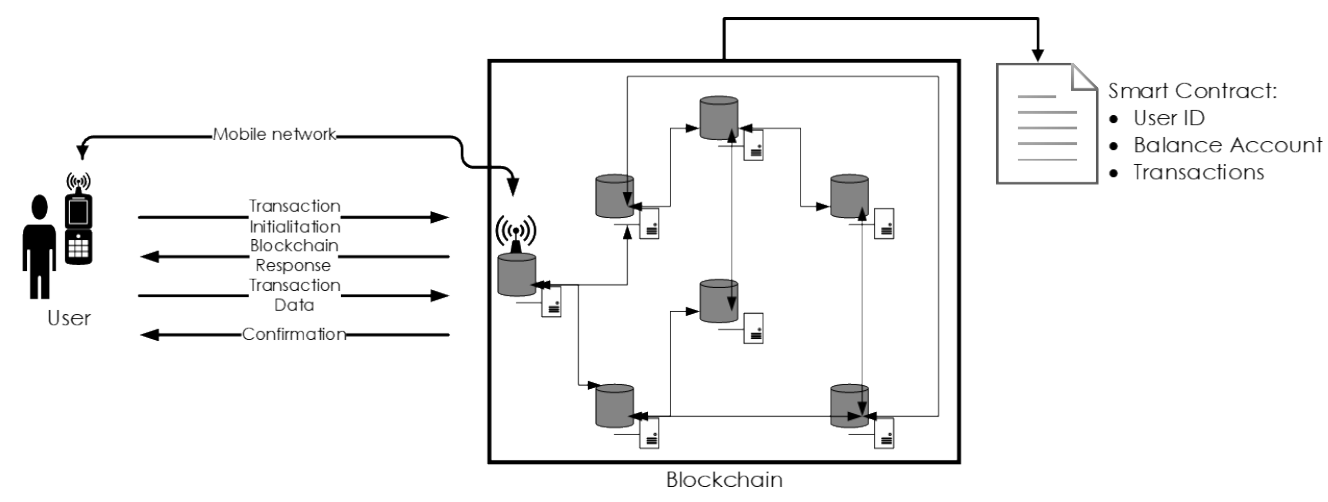

Fig. 1: Offline System Structure

We define a node called Gateway Node to manage the communication between the blockchain network and the end-user device over the mobile network. The Gateway Node acts as a buffer for transactions before being mined. The Gateway node also acts as an enabler of the consensus protocol (in this case the Proof of Stake (PoS) as the new consensus protocol for Ethereum 2.0 network) for the buffered transactions. To maintain the neutrality of the transaction, the Gateway Node starts the consensus but never engages on it. The other nodes, however, execute the PoS when the Gateway Node starts the process.

Every node of the blockchain network must be associated with a bank or a group of banks, that will handle the financial transactions for every user. In particular, the banks will ensure the communications established with its users and create a link between the classical user bank account and the Smart Contract created for the offline transactions model, in a way that is similar to the model described in [28]. Hence, users undergo transactions in both ways (standard face-to-face bank transaction and via SMS) and all operations made to the user's account will be updated on the Smart Contract and vice versa.

\subsection{Smart Contract Features}

To store the information of every user in the Ethereum blockchain, it is important to design a specific Smart Contract, which will also be used to store the balance of the user, and act as a ledger of every transaction made or received by the user. Fig. 2 illustrates the different fields needed by our proposed Smart Contract required to secure and manage all transactions. Each user must be identified by their own Ehtereum direction, based on the account number of their bank accounts, the related PIN and other identification information (such as names). The name will be used to search for the user, as described in the Name search process (Fig. 5a). Besides keeping the balance for the account and an array of transactions made by the users, the Smart Contract will also perform several other functions and operations, such as generating PIN or encrypting the balance and the user's transactions. In Ethereum technology, when the data type address is used in a Smart Contract, it is possible to use embedded balance and transaction functions and to modify them in order to accomplish, not only the security storage, but also the privacy of every user's data within the network. 


\begin{tabular}{|l|}
\hline \multicolumn{2}{|c|}{ Transaction Manager } \\
\hline private address UAddr; \\
\hline private uint uPin; \\
\hline private string UShowName; \\
\hline public double balance; \\
\hline private struct Transaction; \\
\hline public Transaction \\
transactions[]; \\
\hline DEFINE MODIFIERS () \\
\hdashline uPinCalculation() \\
\hdashline balanceHCypher() \\
\hdashline balanceH() \\
\hline transactionH() \\
\hline storeTransaction() \\
\hline
\end{tabular}

Fig. 2: Basic Smart Contract Structure

\subsection{Communication Features}

Without a broadband communication, each transaction exchanged between the user (a cellphone) and the ledger (the Gateway Node) should have two fundamental features:

- A maximum of 140 ASCII characters

- Identifiers and commands that will be easily remembered and entered by a user having a device equipped with a non-QWERTY keyboard.

Taking the two features mentioned above, we define several specific message structures used for the transaction between the users and the Gateway Node, as follows:

- Initialization and Authentication: Fig. 3 illustrates how, in general terms, the transaction scheme is performed. The user usually starts the session by sending an SMS to authenticate himself to the Gateway Node, as the initialization message. This initalization message triggers the authentication phase, explained hereinafter. When authentication is complete (1), the system asks the user to select the operation he wishes to perform (2) with a message to the user as a response for a successful authentication. As an answer to the operation message sent by the gateway, the user send one with the transaction he need to perform (3). Once the transaction/operation is complete, the system ends the connection with the user (4)

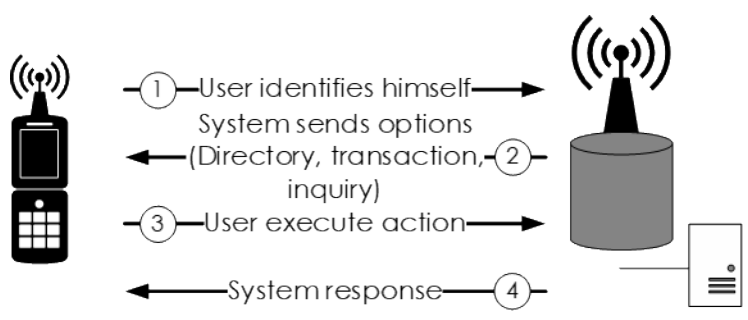

Fig. 3: Offline transactions general scheme 
Fig. 4 specifies how the authentication process is achieved. As an initialization message, the user sends a HELLO-type message, which conveys the user's identity, also known as the PIN to the Gateway Node (1). The blockchain network, which receives the message through the Gateway Node, verifies the existence of the user's PIN and initiates a challenge-response handshake with the user to verify the user's identity, this before starting any subsequent transaction process (2). After the network successfully identifies the user, the Gateway Node will: a) block all initialization attempts from any other device until the transaction ends or or is considered as lost (due to delay, fail of authentication, lost of connection, etc.), and b) send back an ASCII passcode or a random number to the user to authenticate the current connection. As part of the challenge-response handshake, the user must identify himself to the network, by transmitting some personal information, such as a portion of the user's identity number or personal/biometrical validation data in a form of ASCII mnemonic code defined by the Smart Contract(3). This information is unique for every user and work as a passcode/password against the system. As a result, the network will be able to validate the user's identity and start the transactions. When the identification/authentication process is performed successfully, the network, through the Gateway Node, will ask the user to determine the transaction/operation he wishes to perform (4).

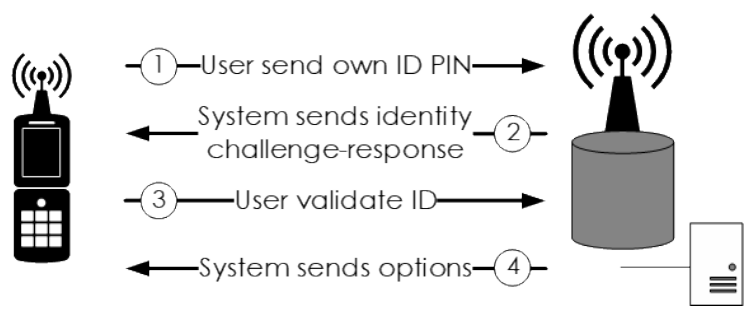

Fig. 4: User authentication process

- Transaction Items: Our system defines three types of transaction operations between the users and the blockchain:

- Name search: It may happen that the users do not know or remember the PIN of the receiver of the transaction initiated by a particular user. In this optic, Fig. 5a illustrates how the blockchain network will implement a Name Search System. Users need to make a request to the blockchain network to start a search for a specific user that will be the destinatary of the transaction (1), and the network will reply by asking for an identifier (e.g., User ID number, User account or User name) for the requested user (2). In (3), the user sends the identifier to the system, that determines whatever the queried user exists (4a) or not (4b) as illustrated in Fig. 5b. The query/response process will continue as long as the user has further transactions; otherwise, the system will close the communication $(5,6,7)$.

- Values Transaction: Once the user has confirmed the transaction recipient's PIN, he will start the transfer of values, as illustrated in Fig. 6a. When the user starts the transaction (1) and receives the confirmation from the system (2), the user shall specify the amount of currency to be transferred, along with the PIN of the receiver. The network will therefore verify and check the transaction before performing it (3). Fig 6b illustrates how the network will validate the received transaction based on the data transmitted by the user. Particularly, it will validate if the ID associated with the requested PIN exists (4a). Then, it will verify if the user has sufficient amount of currency in his/her account to perform the transaction (4b). If both verification processes are achieved successfully, the transaction is then performed and stored using homomorphic encryption [29](4c). Finally, the network will close the connection in case no further transactions are received from the user $(5,6,7)$.

- Inquiry Balance: All the transactions will be supported by Ethereum capabilities to generate balances from the information stored in every Smart Contract. Fig. 7 illustrates how the system will act when it receives a request from the user to ask for the available currency amount in his/her account. When the user asks for the the available balance (1), the system will check the user's Smart Contract and return the balance information (2). After that, the network will close the session in case no further transactions are received from the user $(3,4,5)$. 


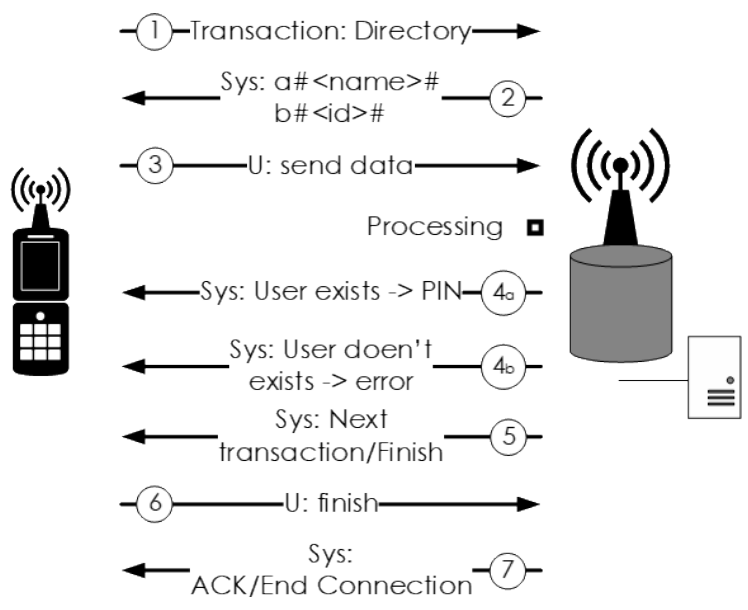

(a) Name search messages

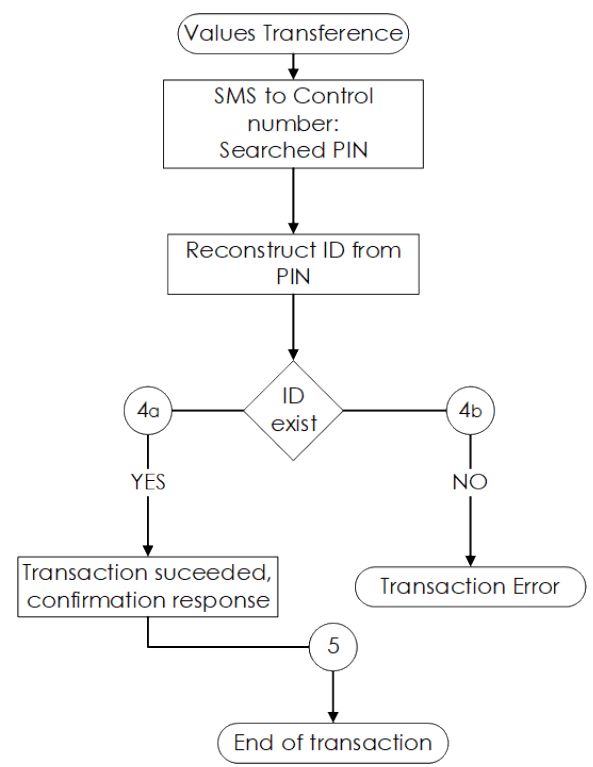

(b) Name search flowchart

Fig. 5: Name search Process

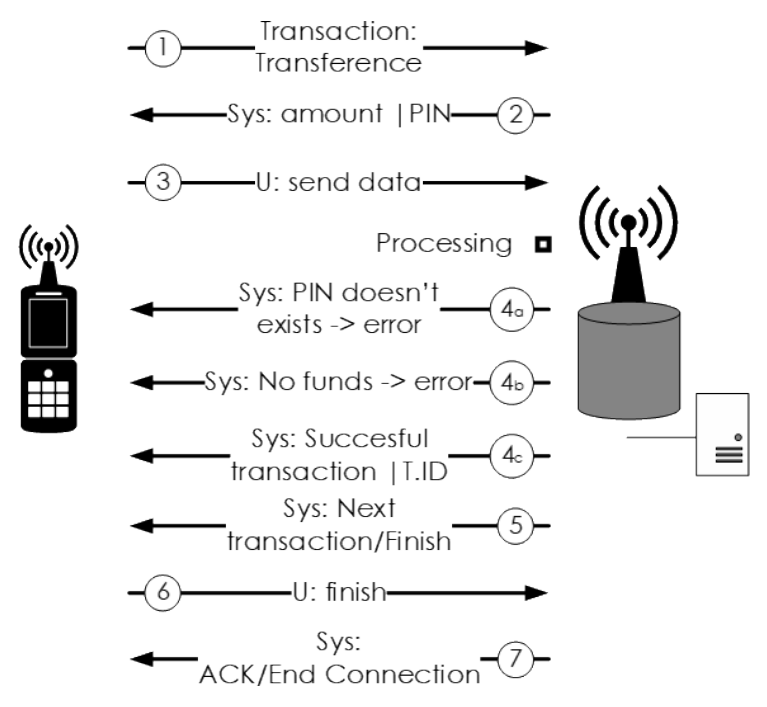

(a) Values transaction messages

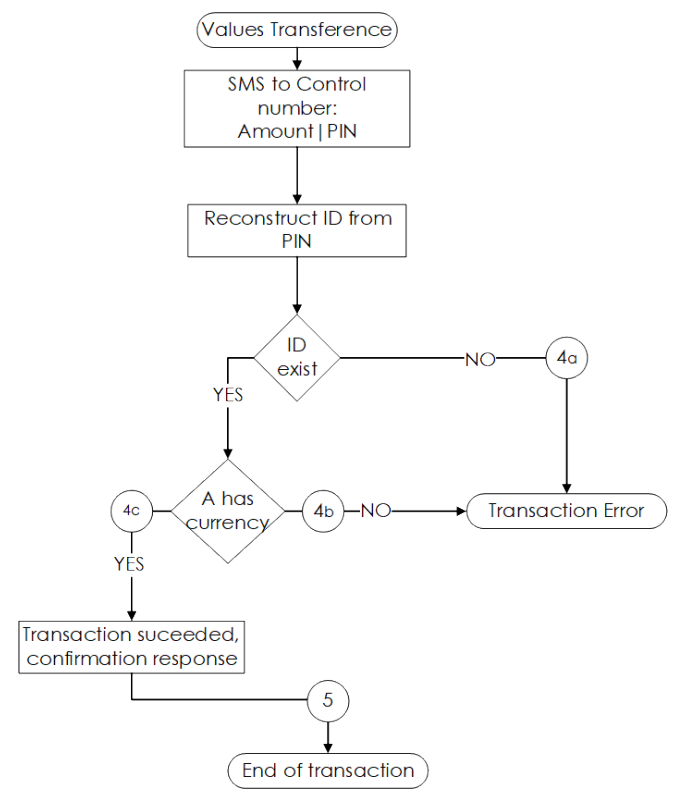

(b) Values transaction flowchart

Fig. 6: Values Transaction Process 


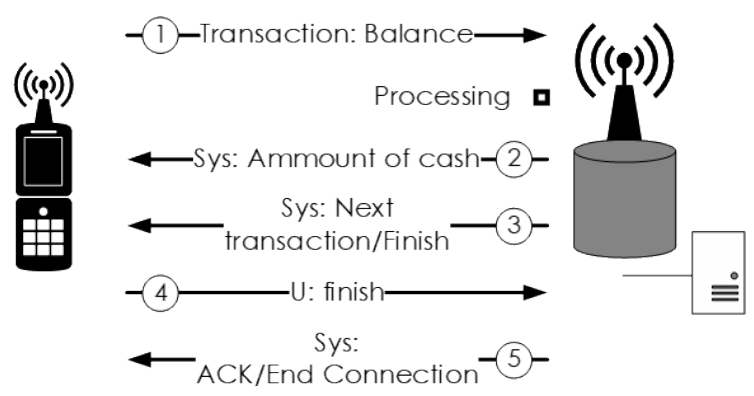

Fig. 7: Inquiry messages

\section{Analysis}

In this section, we present a security analysis of our proposed solution and we evaluate it versus the aforementioned solutions. We also demonstrate its effectiveness in preserving the user's privacy and in maintaining the integrity and confidentiality of data.

\subsection{Blockchain Security Analysis}

The first and most important consideration relies on how transactions are stored in the blockchain. It is obvious that transactions are securely stored in the blockchain. However, it is not possible to manipulate them in their encrypted format and the system needs to decrypt them first, which may cause several security risks. Even with the idea that only the bank which manages the user's bank account can access the ledger records, it is still a security issue. Consequently, our proposed system applies an additive homomorphic cryptosystem on user's transactions to enable computations and financial related functions to be carried out on the encrypted transactions, without revealing the transaction plain text [29]. Consequently, Smart Contracts could execute the programmed operations over the encrypted transactions that are stored in the blockchain without having to individually decrypt each transaction. With this, we intend to solve one of the main security issues of blockchain: privacy on stored data.

\subsection{Identity Management}

Part of our proposal is to break any link between the user's account and the mobile device (or a phone number) that the user might use to connect to the system. Our proposal intends to give the users the flexibility to interact with the system without a specific device. We considered that it is as difficult to have a stable broadband connection as to have a specific device to interact with the system. To have access to the transaction system, every user must connect to the Gateway node through a specific service number (similar to 911 or other short communication numbers) and validate their identity, no matter what phone number the message originates from. In this context, the identity management will be completely managed by our proposed system. The latter provides an easy-to-remember ASCII PIN to identify the user and the PIN is generated based on both the user identity already stored by the financial institutions and the address stored in the Smart Contract.

That identity management paradigm is used because it is hard and insecure for the user to create identity credentials from their mobile phone by using SMSs. Moreover, this paradigm makes it easier for the network to validate and confirm the user identity through a challenge-response mechanism, instead of a password that could be lost or stolen from the user.

\subsection{Offline and delayed transactions}

The main difference between a classical online transaction system and our proposed system is that the transactions will not be executed in broadband real time, but mobile network time as an offline communication. This offline feature may include a variety of delays, both in transit and in message processing on the Gateway. In fact, the transaction that is received from the user and that is to be executed must first of all be 
stored in its corresponding Smart Contract. Hence, the transaction should first be mined before updating both the user account and the Smart Contract to consolidate the information. Once the user transaction is executed by the system, the user will receive a confirmation of the transaction. In other words, and to avoid any double-spending, the system is designed to lock the current session and consolidate the legder before any transaction confirmation is sent to the user.

Some delay may depend on the time needed by the mobile network to transmit all messages related to a transaction. Although this delay cannot be considered as a security breach, it should be taken into account to find ways to handle it, so global transaction time will be reduced and the involved parties (users and the network) can improve their response times for every transaction.

The delay between the time a user sends a transaction message and the time the same user receives a confirmation could be critical and needs to be short enough to emulate an online transaction performance. But, even with all the possible improvements, all the proposed transactions are still in mobile band time; which means that the whole system needs to take into account different keep-alive session times rather than the broadband ones. Consequently, a specific time window is defined for our proposed system to keep the session alive in harsh connection conditions. That window window considers all the delays in the classical mobile network communications to define the border times in order to receive a message during the established session. In other words, the time needed to consider a session lost or expired is larger than in other transaction over a broadband connection. For as long as a session can last, the user PIN will be blocked to start a new session. That way, the user does not need to re establish a new session or lose transactions because of mobile network delays or coverage issues.

\subsection{Mobile operator role}

In the context of our proposed system, the mobile network will be only used as a communication channel, so the mobile network is not involved in the creation or processing of the user information. However, the system must ensure that the network supports the message format and character codification for all mobile devices. The main idea of this proposal is to run bank transactions in harsh environments, where SMS is the only available way to exchange data between the user and the system.

\subsection{Comparison}

Table 1: Comparison table between existent solutions and this proposal

\begin{tabular}{|c|c|c|c|c|c|c|c|c|c|c|}
\hline $\begin{array}{c}\text { Parameters } \\
\text { Works }\end{array}$ & $\begin{array}{c}\text { Offline } \\
\text { Access } \\
(1)\end{array}$ & $\begin{array}{c}\text { Secure } \\
\text { Storage } \\
(2)\end{array}$ & $\begin{array}{c}\text { Transmission } \\
\text { Media } \\
(3)\end{array}$ & $\begin{array}{c}\text { Identity } \\
\text { Privacy } \\
(4)\end{array}$ & $\begin{array}{c}\text { Identity } \\
\text { Generation } \\
(5)\end{array}$ & $\begin{array}{c}\text { Secure } \\
\text { Transaction } \\
(6)\end{array}$ & $\begin{array}{c}\text { Broadband } \\
\text { Connection } \\
(7)\end{array}$ & $\begin{array}{c}\text { Users } \\
\text { as Nodes } \\
(8)\end{array}$ & $\begin{array}{c}\text { App } \\
\text { Requirement } \\
(9)\end{array}$ & $\begin{array}{c}\text { Tokens } \\
\text { as Money } \\
(10)\end{array}$ \\
\hline $\begin{array}{c}\text { Our } \\
\text { Proposal }\end{array}$ & Yes & Yes & $\begin{array}{c}\text { Open } \\
\text { Wireless }\end{array}$ & No & $\begin{array}{c}\text { Via } \\
\text { App }\end{array}$ & Yes & Mandatory & Yes & Yes & Yes \\
\hline
\end{tabular}

Table 1 provides a theoretically comparison between our proposed solution and the solution proposed in [11] in terms of the following features:

- Network Access $(1,3,7)$ : how users interact with the blockchain network and the compared connection capabilities.

- Security $(2,4,6)$ : This includes features offered by the network to ensure secure data storage, authentication and privacy of all user information and interactions.

- Identity Management (5): How users can access to an identity to use and manipulate the network and their own stored information.

- Users Interaction $(8,9)$ : How users can communicate with the network and what types of devices they need to start the transactions.

- Representation of currency (10): How the network understands the units of currency and manages them for every transaction. 
The above features guide us to compare our proposed solution to the solution described in [11]. It is worth noting that the features (1)(3)(7) in Table 1 show that users using our proposal don't need a broadband connection to interact with the network, which is not the case of the solution in [11].

The features (2)(4)(6) in Table 1 demonstrate that our proposal intends to manage the information stored in the Smart Contract, by cypher the user transactions as well as the user's identity information, so that the user's data is stored in a secure and private way. Feature (5) shows how the users' identities are managed in both works. In our proposal, the identity is created by the bank and the interaction between the bank account and the information in the Smart Contract will create the user PIN. Instead, the other solution relies on the user's device to create the user's identity through and defined app. As we discussed in Section 4.2, it is more secure to let the banks manage their users' identities, rather than assigning this to any third party. Features (8) and (9) compare the interaction between the users and the network, and illustrate that our proposal does not need any app to communicate with the users. Consequently, the users can not be network nodes, where in the solution discussed in [11], a user can be a network node. Finally, feature (10) shows how both proposals manage the currency. In fact, our proposal uses the same currency used by the bank for transactions and balances, and hence, the transparency of transactions between the network, the bank and the users is guaranteed. However, the solution in [11] converts currency into token and consequently, its use is limited to few banks supporting these type of tokens.

\section{Conclusion and Future Work}

In this paper, we present and analyse an end-to-end and low overhead secure solution for the communications between limited connectivity mobile devices and their corresponding remote applications servers using blockchain. Our system incorporates the deployment of Ethereum network and its Smart Contracts and extends it with internal encryption to make the information not only secure and tamper-resistant but also private for every user. Financial information is considered very sensitive for all the regulation authorities in the world and keeping that information secure is not enough to have a whole secure banking system. With our proposal, we not only intend to create a way to transact with banks offline but also to map between the Smart Contract and the classical bank accounts to improve banking management and services. Our proposed solution intends to create and accessible system for every bank user and to keep their transactions accessible, transparent and secure. Our future work consists in implementing our proposed solution using Solidity, which is an object-oriented, high-level language for implementing smart contracts. In addition, we are planning to implement the infrastructure that allows the Gateway Node to manage transactions between mobile users and their banking systems through the blockchain in a way that mobile transaction delays can be reduced to improve the speed of all transactions.

\section{References}

1. Nakamoto, S.: Bitcoin: A peer-to-peer electronic cash system (2008), https://bitcoin.org/bitcoin.pdf

2. Maldonado-Ruiz, D., Torres, J., El Madhoun, N.: 3bi-ecc: a decentralized identity framework based on blockchain technology and elliptic curve cryptography. 2020 2nd Conference on Blockchain Research \& Applications for Innovative Networks and Services (BRAINS) pp. 45-46 (2020)

3. Wood, G.: Ehtereum: A Secure Decentralized Generalized Transaction Ledger. Ethereum Project Yellow Paper pp. 1-32 (2018), https://www.cryptopapers.info/eth_yellow

4. Eyal, I.: Blockchain Technology: Transforming Libertarian Cryptocurrency Dreams to Finance and Banking Realities. Computer 50(9), 38-49 (2017). https://doi.org/10.1109/MC.2017.3571042, http://ieeexplore.ieee.org/document/8048646/

5. Bello, G., Perez, A.J.: Adapting Financial Technology Standards to Blockchain Platforms. In: Proceedings of the 2019 ACM Southeast Conference on ZZZ - ACM SE '19. pp. 109-116. ACM Press, New York, New York, USA (2019). https://doi.org/10.1145/3299815.3314434, http://dl.acm.org/citation.cfm?doid=3299815.3314434

6. Xu, Z., Wang, Q., Wang, Z., Liu, D., Xiang, Y., Wen, S.: PPM: A Provenance-Provided Data Sharing Model for Open Banking via Blockchain. In: Proceedings of the Australasian Computer Science Week Multiconference. pp. 1-8. ACM, New York, NY, USA (feb 2020). https://doi.org/10.1145/3373017.3373022, https://dl.acm.org/doi/10.1145/3373017.3373022 
7. Oktian, Y.E., Witanto, E.N., Kumi, S., Lee, S.G.: BlockSubPay - A Blockchain Framework for SubscriptionBased Payment in Cloud Service. International Conference on Advanced Communication Technology, ICACT 2019-February, 153-158 (2019). https://doi.org/10.23919/ICACT.2019.8702008

8. Bhaskaran, K., Ilfrich, P., Liffman, D., Vecchiola, C., Jayachandran, P., Kumar, A., Lim, F., Nandakumar, K., Qin, Z., Ramakrishna, V., Teo, E.G., Suen, C.H.: Double-Blind Consent-Driven Data Sharing on Blockchain. In: 2018 IEEE International Conference on Cloud Engineering (IC2E). pp. 385-391. IEEE (apr 2018). https://doi.org/10.1109/IC2E.2018.00073, https://ieeexplore.iee.org/document/8360358/

9. Kafshdar Goharshady, A., Behrouz, A., Chatteriee, K.: Secure Credit Reporting on the Blockchain. In: 2018 IEEE International Conference on Internet of Things (iThings) and IEEE Green Computing and Communications (GreenCom) and IEEE Cyber, Physical and Social Computing (CPSCom) and IEEE Smart Data (SmartData). pp. 1343-1348. IEEE (jul 2018). https://doi.org/10.1109/Cybermatics_2018.2018.00231, https://ieeexplore.ieee.org/document/8726769/

10. Selvaraj, P., Prabakaran, S., Krishnateja, V.: Novel Payment Wallet Management with Blockchain Based Cryptocurrency. International Journal of Recent Technology and Engineering 8(2S4), 228-233 (aug 2019). https://doi.org/10.35940/ijrte.B1042.0782S419, https://www.ijrte.org/wpcontent/uploads/papers/v8i2S4/B10420782S419.pdf

11. Hu, Y., Manzoor, A., Ekparinya, P., Liyanage, M., Thilakarathna, K., Jourjon, G., Seneviratne, A.: A Delay-Tolerant payment scheme based on the ethereum blockchain. IEEE Access 7, 33159-33172 (2019). https://doi.org/10.1109/ACCESS.2019.2903271

12. Zhang, R., Xue, R., Liu, L.: Security and Privacy on Blockchain. ACM Computing Surveys 52(3), 1-34 (jul 2019). https://doi.org/10.1145/3316481, http://dl.acm.org/citation.cfm?doid=3341324.3316481

13. Ahmed, M., Elahi, I., Abrar, M., Aslam, U., Khalid, I., Habib, M.A.: Understanding Blockchain. In: Proceedings of the 3rd International Conference on Future Networks and Distributed Systems - ICFNDS '19. pp. 1-8. ACM Press, New York, New York, USA (2019). https://doi.org/10.1145/3341325.3342033, http://dl.acm.org/citation.cfm?doid=3341325.3342033

14. Narayanan, A., Clark, J.: Bitcoin's academic pedigree. Communications of the ACM 60(12), 36-45 (nov 2017). https://doi.org/10.1145/3132259, http://dl.acm.org/citation.cfm?doid=3167461.3132259

15. Dorri, A., Kanhere, S.S., Jurdak, R., Gauravaram, P.: Blockchain for IoT security and privacy: The case study of a smart home. In: 2017 IEEE International Conference on Pervasive Computing and Communications Workshops (PerCom Workshops). pp. 618-623. IEEE (mar 2017). https://doi.org/10.1109/PERCOMW.2017.7917634, https://ieeexplore.ieee.org/document/7917634/

16. Kravitz, D.W., Cooper, J.: Securing user identity and transactions symbiotically: IoT meets blockchain. In: 2017 Global Internet of Things Summit (GIoTS). pp. 1-6. IEEE, Geneva, Switzerland (jun 2017). https://doi.org/10.1109/GIOTS.2017.8016280, http://ieeexplore.iee.org/document/8016280/

17. Bragagnolo, S., Marra, M., Polito, G., Gonzalez Boix, E.: Towards scalable blockchain analysis. In: 2019 IEEE/ACM 2nd International Workshop on Emerging Trends in Software Engineering for Blockchain (WETSEB). pp. 1-7 (May 2019). https://doi.org/10.1109/WETSEB.2019.00007

18. Yue, L., Junqin, H., Shengzhi, Q., Ruijin, W.: Big data model of security sharing based on blockchain. In: 2017 3rd International Conference on Big Data Computing and Communications (BIGCOM). pp. 117-121 (Aug 2017). https://doi.org/10.1109/BIGCOM.2017.31

19. BANDARA, E., NG, W.K., DE ZOYSA, K., FERNANDO, N., THARAKA, S., MAURAKIRINATHAN, P., JAYASURIYA, N.: Mystiko-blockchain meets big data. In: 2018 IEEE International Conference on Big Data (Big Data). pp. 3024-3032 (Dec 2018). https://doi.org/10.1109/BigData.2018.8622341

20. Tewari, H., Hughes, A., Weber, S., Barry, T.: X509Cloud - Framework for a ubiquitous PKI. In: MILCOM 2017 - 2017 IEEE Military Communications Conference (MILCOM). vol. 2017-Octob, pp. 225-230. IEEE, Baltimore, MD, USA (oct 2017). https://doi.org/10.1109/MILCOM.2017.8170796, http://ieeexplore.ieee.org/document/8170796/

21. Karaarslan, E., Adiguzel, E.: Blockchain Based DNS and PKI Solutions. IEEE Communications Standards Magazine 2(3), 52-57 (sep 2018). https://doi.org/10.1109/MCOMSTD.2018.1800023, https://ieeexplore.ieee.org/document/8515149/

22. Axon, L., Goldsmith, M.: PB-PKI : a Privacy-Aware Blockchain-Based PKI. In: Proceedings of the 14th International Joint Conference on e-Business and Telecommunications (ICETE 2017). vol. 4, pp. 311 - 318. SciTePress, Madrid, Spain (2017), https://www.scopus.com/inward/record.uri?eid=2-s2.085029461108\&partnerID $=40 \& m d 5=a 3 c 5 b d 3 c 1 f 35085 a 047453 a d 1 c 386 c 9 c$

23. Won, J., Singla, A., Bertino, E., Bollella, G.: Decentralized Public Key Infrastructure for Internetof-Things. In: MILCOM 2018 - 2018 IEEE Military Communications Conference (MILCOM). pp. 907-913. IEEE, Los Angeles, CA, USA (oct 2018). https://doi.org/10.1109/MILCOM.2018.8599710, https://ieeexplore.ieee.org/document/8599710/ 
24. Shahzad, B., Crowcroft, J.: Trustworthy Electronic Voting Using Adjusted Blockchain Technology. IEEE Access 7, 24477-24488 (2019). https://doi.org/10.1109/ACCESS.2019.2895670, https://ieeexplore.ieee.org/document/8651451/

25. Carr, L., Newtson, A.J., Joshi, J.: Towards Modernizing the Future of American Voting. In: 2018 IEEE 4th International Conference on Collaboration and Internet Computing (CIC). pp. 130-135. IEEE (oct 2018). https://doi.org/10.1109/CIC.2018.00028, https://ieeexplore.ieee.org/document/8537826/

26. Hanifatunnisa, R., Rahardjo, B.: Blockchain based e-voting recording system design. In: 2017 11th International Conference on Telecommunication Systems Services and Applications (TSSA). pp. 1-6. IEEE (oct 2017). https://doi.org/10.1109/TSSA.2017.8272896, http://ieeexplore.ieee.org/document/8272896/

27. Harris, C.G.: The risks and dangers of relying on blockchain technology in underdeveloped countries. In: NOMS 2018 - 2018 IEEE/IFIP Network Operations and Management Symposium. pp. 1-4. IEEE, Taipei, Taiwan (apr 2018). https://doi.org/10.1109/NOMS.2018.8406330, https://ieeexplore.ieee.org/document/8406330/

28. Peters, G.W., Panayi, E.: Understanding Modern Banking Ledgers Through Blockchain Technologies: Future of Transaction Processing and Smart Contracts on the Internet of Money. In: Banking Beyond Banks and Money, pp. 239-278 (2016). https://doi.org/10.1007/978-3-319-42448-4_13, http://link.springer.com/10.1007/978-3-31942448-4 http://link.springer.com/10.1007/978-3-319-42448-4_ 13

29. Fontaine, C., Galand, F.: A survey of homomorphic encryption for nonspecialists. EURASIP Journal on Information Security 2007, 1-10 (2007) 